\title{
Treatment response among treatment naïve non cirrhotic chronic hepatitis c patients with triple therapy: A study from Punjab (India)
}

\begin{abstract}
Introduction: India has a low to moderate prevalence of Hepatitis C Virus (HCV) infection $(0.09 \%-2.02 \%)$. However, in a recent population based study from Punjab, the HCV prevalence rate of $5.2 \%$ was found. The biggest milestone was achieved in March 2015, when Sofosbuvir (SOF) rolled in the Indian market. In this article, we present the results of triple therapy (Peg-Interferon (PEG-INF)+Sofosbuvir (SOF)+Ribavirin (RBV) among treatment naive Chronic Hepatitis C patients.
\end{abstract}

Material and Methods: Medical records of all the patients who were diagnosed to be treatment naïve chronic hepatitis $\mathrm{C}(\mathrm{HCV})$ and who received Triple Therapy treatment (Peg INF $\alpha 2 \mathrm{a} / \alpha 2 \mathrm{~b}+$ Ribavirin (RBV)+Sofosbuvir (SOF) between March 2015 and Aug 2015 were analyzed. Detailed history, physical examination and laboratory investigations were done at regular interval. HCV-RNA was repeated at 4th, 8th, 12 thweek and 12 weeks after the end of therapy. Sustained virological response at 12 weeks (SVR-12) was noted for all patients, in whom it was available.

Results: A total of 84 patients were included in the study. Male patients dominated in the study group [61 male (72.6\%) \& 23 females $(27.3 \%)] .38$ Patients $(45.2 \%)$ belonged to $<40 \mathrm{yrs}$ age group while $46(54.7 \%)$ patients were $>40 \mathrm{yrs}$ of age. Genotype 3 was the most common genotype detected in our patients [59(70.2\%)] followed by genotype $1[20(23.8 \%)]$ and genotype 4 patient $[5(5.9 \%)]$. HCV RNA was undetected at 4 th week in $78(92.85 \%)$ while End of therapy response (ETVR) was seen in $80(95.23 \%)$ patients. Sustained virological response (SVR) at 12 thweek was seen in $73(86.90 \%)$ patients, 6 patients were lost to follow-up (LFU) while 5 did not achieve SVR. None of the patients had significant adverse effects requiring termination of therapy. History of IV drug abuse indicating this as the likely source of HCV was obtained in 15 patients $(17.8 \%$ ). History of $\mathrm{HCV}$ in one or more family member (first degree relative) was present in 10 patients $(11.9 \%)$.

Conclusion: With the introduction of sofosbuvir (SOF), a pan genomic directly acting antiviral (DAA) drug, in the treatment (triple therapy) protocol, not only the duration of therapy and side effects has decreased but even SVR has shown a rise. With the advent of equally/more effective interferon free regimens, treatment among chronic hepatitis $\mathrm{C}$ patients is bound to see a revolution.

Keywords: sofosbuvir, triple therapy, HCV infection, aminotransferases, drug abuse, fibroscan, HCV seroprevalence, SVR rates, DAA drug, hepatitis c genotype
Volume 4 Issue I - 2018

Amit Soni, Nirmaljeet Singh Malhi

Department of Gastroenterology and Liver Diseases, SPS

hospital, India

Correspondence: Nirmaljeet S Malhi, Department of Gastroenterology and Liver Diseases, SPS Hospital, Sherpur Chownk, Ludhiana, India, Tel 9I-88 72054262. Email drnjsmalhi@gmail.com

Received: December 18, 2017 | Published: February 01, 2018
Abbreviations: CLD, chronic liver disease; HCV, hepatitis c virus; SOF, sofosbuvir; SVR, sustained virological response; ETVR, end of therapy response; LFU, lost to follow-up; DAAs, directly acting anti-virals; CBC, complete blood count; LFT, liver function test; INR, international normalized ratio; USG, ultrasound; RFT, renal function test; SVR-12, sustained virological response; ETR, end of treatment response

\section{Introduction}

Chronic hepatitis $\mathrm{C}$ remains asymptomatic in initial stages in majority of patients. Moreover untreated chronic hepatitis $\mathrm{C}$ increases the risk of Cirrhosis of liver, liver failure and hepato cellular carcinoma. ${ }^{1}$

India has a low to moderate prevalence of Hepatitis C Virus (HCV) infection $(0.09 \%-2.02 \%)$ but still accounts for a significant share of
HCV infection worldwide due to its large population. ${ }^{2}$ The Punjab state needs a special mention as the prevalence of $\mathrm{HCV}$ infection has been reported to be much higher when compared to the rest of India. In a recent population based study from Punjab, where in a house to house survey was conducted a prevalence rate of $5.2 \%$ was found. ${ }^{3}$ Some area of Punjab have the prevalence much above this reported prevalence rate. ${ }^{4}$ This exceptionally high prevalence has been attributed by the experts to growing number of injectable drugs uses in the state and wrong injection practices over last few decades. Even worse, in a study, it was seen that $18 \%$ doctors were still reusing needles and syringes. ${ }^{5}$

Innovation in the field of Hepatitis $\mathrm{C}$ treatment has been expeditive, and decisive. Interferon free regimen has engaged the western market since long. The new drugs are not just more safe and acceptable to patients but they have further unfolded the patient groups in whom these can be used. The biggest milestone was achieved in March 2015, 
when Sofosbuvir (NS5B inhibitor) rolled in the Indian market at very affordable price when compared to west (1000\$ a pill). ${ }^{6}$

SOF is an oral nucleotide analogue inhibitor of HCV specific NS5B polymerase. SOF has the pangenomic effectiveness and has a high genetic barrier to development of viral resistance. ${ }^{7}$ Many more Directly Acting Anti-virals (DAAs) like Ledipasvir and Daclatasvir are expected to roll in Indian Market shortly and at a fair cost. Triple therapy i.e. SOF with PEG-INF and RBV has been successfully used in numerous trials for genotype 1, 3 and 4 . Though it is not needed in genotype 2 where dual therapy (SOF and RBV) has shown promising results. The supremacy of triple therapy over PEG-IFN and RBV is due to owed to numerous advantages that it provides. The benefits include shorter duration of therapy (12weeks), superior SVR, less adverse events and higher patient's acceptability. ${ }^{8}$ In this article, we present the results of triple therapy (PEG-INF+SOF+RBV) among previously untreated Chronic Hepatitis $\mathrm{C}$ patients.

\section{Material and methods}

Medical records of all the patients who were diagnosed to be treatment naïve chronic hepatitis $\mathrm{C}$ and who received Triple Therapy treatment (PEG-INF+RBV+SOF) between March 2015 and Aug 2015 were analyzed.

Baseline work up of patients included- complete blood count (CBC), Liver Function Test (LFT) \{Total Bilirubin, SGOT, SGPT, S.albumin, S. Alkaline Phosphatase\}, Renal Function Test (RFT) \{Blood urea, S. Creatinine, S. Sodium, S. Potassium\}, International Normalised Ratio (INR), Ultrasound (USG) abdomen, Upper Gastro Intestinal Endoscopy(UGI), Fibroscan, HCV-RNA (Quantitative and Genotype), HBs Ag and HIV.

\section{Patients with following conditions were excluded:}
a. HBV (Hepatitis B) infection/ HIV disease.
b. Chronic kidney disease.
c. Chronic liver disease other than HCV infection.
d. Malignancy of any body part.

Patients received PEG-INF $\alpha 2 \mathrm{a}$ with oral RBV and SOF. The duration of treatment was 12 weeks for genotype 3,1 and 4. Patients were followed up on outpatient basis weekly for first 4weeks and then every 2 nd week till the end of the therapy. Detailed history, physical examination with CBC, RFT and LFT were maintained. HCV-RNA was repeated at 4th, 8th, 12thweek and 12th weeks after the end of therapy. RBV dose was decreased if $\mathrm{Hb}$ dropped to less than $10 \mathrm{gm} / \mathrm{dL}$ and was discontinued if it further fell $<8.5 \mathrm{gm} / \mathrm{dL}$.

\section{Treatment response was defined as following:}

i. End of treatment response (ETR) is defined as no detectable HCV-RNA in serum and normalization of aminotransferases at completion of treatment.

ii. Sustained Virological Response (SVR-12) is defined as absence of HCV-RNA 12 weeks after the end of therapy.

iii. Non-responder is defined as detectable HCV-RNA at the end of therapy.

iv. Relapser.

The primary endpoint was SVR-12. Statistical analyses was done using SPSS version 18 (Intention to treat analysis was used).

\section{Results}

A total of 84 patients were included in the study. Male patients dominated in the study group [61 male (72.6\%) \& 23 females (27.3\%)] (Figure 1). 38 patients (45.2\%) belonged to $<40 \mathrm{yrs}$ age group while $46(54.7 \%)$ patients were $>40 \mathrm{yrs}$ of age. Genotype 3 was the most common genotype detected in our patients [59(70.2\%)] followed by genotype $1[20(23.8 \%)]$ and genotype 4 patient [5 (5.9\%)] (Figure $2)$. Majority of patients $[50(59.5 \%)]$ had viral load $>6$ lac $\mathrm{IU} / \mathrm{mL}$ while the remaining $34(40.5 \%)$ patients had $<6$ lakh HCV RNA IU/ $\mathrm{mL}$ (Figure 3). HCV RNA was undetected at 4th week in 78(92.85\%) while ETVR was seen in $80(95.23 \%)$ patients. SVR at 12 thweek was seen in $73(86.90 \%)$ patients, 6 patients were LFU while 5 did not achieve SVR.

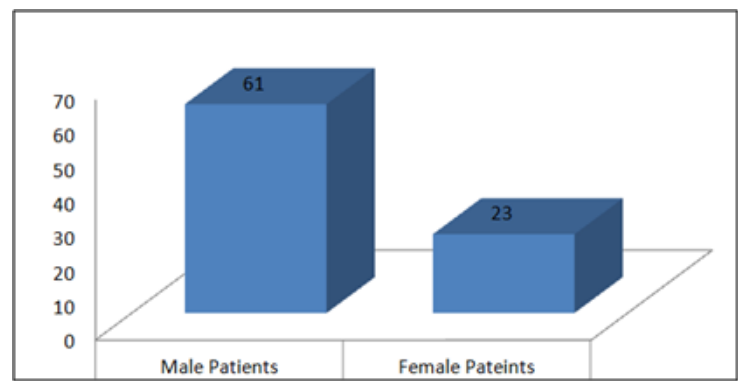

Figure I Figure showing number of male and female patients respectively.

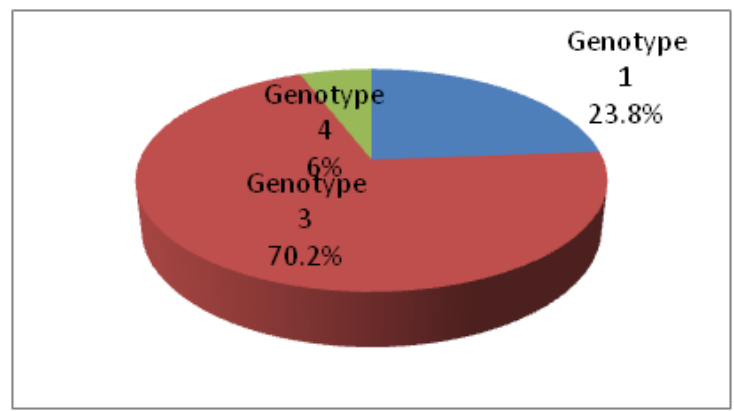

Figure 2 Figures showing percentage of various HCV genotypes seen among chronic hepatits $\mathrm{C}$ patients.

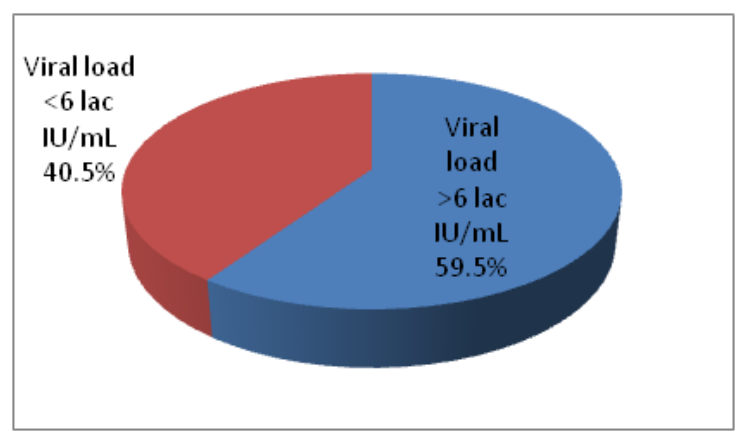

Figure 3 Figure showing distribution of $\mathrm{HCV}$ viral load among chronic hepatitis $C$ patients.

None of the patients had significant adverse effects requiring termination of therapy. $8(9.5 \%)$ patients had $\mathrm{Hb}<9 \mathrm{gm} / \mathrm{dL}$ at one or the other period of time during therapy. Other adverse effects included myalgia, fever, weight loss and rashes. History of IV drug abuse indicating this as the likely source of $\mathrm{HCV}$ was obtained in $15(17.8 \%)$ patients. History of $\mathrm{HCV}$ in one or more family member (first degree relative) was present in $10(11.9 \%)$ patients. Fibroscan was performed in all patients while liver biopsy was performed in none. 


\section{Discussion}

In our study group, male patients constituted nearly three fourth of the population. In a study on hepatitis $\mathrm{C}$ patient from Punjab a similar percentage was seen wherein male population constituted $68.4 \%$ of the study group. ${ }^{9}$

In India, it has been reported in many studies that genotype 3 is the predominant genotype among all. Though a study of Valliammai $\mathrm{T}$ et al. ${ }^{10}$ from south India has reported Genotype 1 as the commonest cause whereas study of Chakravarti A et al. ${ }^{11}$ from North India invariably concluded genotype 3 to be the commonest. In our study too, genotype 3 was the commonest followed by genotype 1 and 4 respectively.

A 10year study of Christdas $\mathrm{J}$ et al. ${ }^{12}$ in hepatitis $\mathrm{C}$ genotype among Chronic Liver Disease (CLD) patients in a tertiary care centre in South India found that genotype 3 was most predominant $(63.85 \%)$ followed by genotype $1(25.72 \%)$, genotype $4(7.5 \%)$, genotype $6(2.7 \%)$ and genotype $2(0.002 \%)$.

In a similar study of Singh $\mathrm{S}$ et al. ${ }^{13}$ genotype 3 was found in $66.6 \%$ of patients followed by genotype 1(13.8\%) and genotype $2(5.5 \%)$ and $4(2.7 \%) .{ }^{13}$ Transmission by IV drug abuse is well known for HCV. A community based study for anti HCV seroprevalence among IVDUs (IV Drug Users) showed an increase from $17 \%$ to $80 \%$ over a 7year period despite ongoing awareness programm. ${ }^{14}$ Also very high prevalence of HCV infection among IVDUs has been reported from north eastern states. ${ }^{15,16} \mathrm{IV}$ drug abuse as the likely cause was elicited in $17.8 \%$ of our patients.

Treatment with dual therapy (PEG-INF and RBV) for HCV is well known to have varied response depending on the genotype. Study by Amarapurkar et al. ${ }^{17}$ concluded that genotype 3 is associated with better treatment response when compared to genotype 1. Overall the sustained treatment response was significantly greater in patients with genotype 3 patients (87.5\%) vs genotype 1(56.2\%). In a similar study of Kumar D, et al. ${ }^{18}$ showed SVR rates were higher for genotype $3(76.6 \%)$ when compared to genotype $1(66.6 \%)$.

With the introduction of SOF, a pan genomic DAA drug, in the treatment protocol, not only the duration of therapy has decreased but even SVR has shown a rise. This is especially true for genotype 1 where SVR used be very low and therapy used is for a minimum of 48weeks. ${ }^{19}$ In patients with HCV genotype-1 (treatment naive, chronic hepatits C), SVR was achieved by $89 \%(95 \%$ CI $77-96)$ patients who were treated with PEG-INF, SOF and RBV for 12weeks duration. ${ }^{20}$ In BOSON Trial, treatment naïve patients with genotype 3 who were given triple therapy had SVR 12 of $95 \%(89$ out of 95 patient $) .{ }^{21}$

\section{Conclusion}

The treatment of chronic hepatitis $\mathrm{C}$ patients (treatment naïve, non cirrhotics) with triple therapy is very promising. With the introduction of SOF, a pan genomic directly acting antiviral (DAA) drug, in the treatment (triple therapy) protocol, not only the duration of therapy and side effects has decreased but even SVR has shown a rise. With the advent of equally/more effective interferon free regimens, treatment among chronic hepatitis $\mathrm{C}$ patients is bound to see a revolution. Genotype 3 remains the commonest genotype in this region. IV drug abuse is responsible for nearly $1 / 5$ th of all cases of hepatitis $\mathrm{C}$.

\section{Acknowledgements}

None.

\section{Conflict of interest}

Author declares that there is no conflict of interest.

\section{References}

1. Toshikuni N, Arisawa T, Tsutsumi M. Hepatitis C-related liver cirrhosis-strategies for the prevention of hepatic decompensation, hepatocarcinogenesis, and mortality. World $J$ Gastroenterol. 2014;20(11):2876-2887.

2. Mukhopadhyaya A. Hepatitis C in India. J Biosci. 2008;33(4):465-473.

3. Sood A, Sarin SK, Midha V, et al. Prevalence of hepatitis C virus in a selected geographical area of northern India: a population based survey. Indian J Gastroenterol. 2012;31(5):232-236.

4. Arora D, Jindal N, Dang R, et al. Rising Seroprevalence of HCV a Silent Killer-Emerging Problem. Int J Pharm Pharm Sci. 2011;3(4):57-59.

5. Sood A, Midha V, Awasthi G. Hepatitis C: Knowledge and practices among the family physicians. Trop Gastroenterol. 2002;23(4):198-201.

6. Dhiman RK. Future of therapy for Hepatitis C in India: A Matter of Accessibility and Affordability? J Clinic \& Exp Hepatol. 2014;4(2):85-86.

7. Gallego I, Sheldon J, Morena E, et.al Barrier-Independent, FitnessAssociated Differences in Sofosbuvir Efficacy against Hepatitis C Virus. Antimicrob. Antimicrob Agents Chemother. 2016;60(6):3786-3793.

8. Palumbo E. Pegylated interferon and ribavirin treatment for hepatitis $\mathrm{C}$ virus infection. Ther Adv Chronic Dis. 2011;2(1):39-45.

9. Sood A, Midha V, Sood N, et al. Pegylated interferon alfa $2 \mathrm{~b}$ and oral ribavirin in patients with HCV-related cirrhosis. Indian J Gastroenterol. 2006;25(6):283-285

10. Valliammai T, Thyagarajan SP, Zuckerman AJ, et al. Diversity of genotypes of hepatitis C virus in southern India. J Gen Virol. 1995;76(Pt 3):711-716.

11. Chakravarti A, Dogra G, Verma V, et al. Distribution pattern of $\mathrm{HCV}$ genotypes \& its association with viral load. Indian J Med Res. 2011;133:326-331.

12. Christdas J, Sivakumar J, David J, et al. Genotypes of hepatitis C virus in the Indian sub-continent: A decade-long experience from a tertiary care hospital in South India. Indian J Med Microbiol. 2013;31(4):349-353.

13. Singh S, Malhotra V, Sarin SK. Distribution of hepatitis C virus genotypes in patients with chronic hepatitis $\mathrm{C}$ infection in India. Indian $J$ Med Res. 2004;119(4):145-148.

14. Sarkar K, Mitra S, Bal B, et al. Rapid spread of hepatitis C and needle exchange programme in Kolkata, India. Lancet. 2003;361(9365):1301-1302.

15. Devi KS, Singh NB, Mara J, et al. Seroprevalence of hepatitis B virus and hepatitis $\mathrm{C}$ virus among hepatic disorders and injection drug users in Manipur - a preliminary report. Indian $J$ Med Microbiol. 2004;22(2):136-137.

16. Das HK, Borkokty BJ, Mahanta $\mathrm{J}$, et al. Hepatitis $\mathrm{C}$ virus infection and risk behaviors among injection drug users in Nagaland. Indian $J$ Gastroenterol. 2007;26:253-254.

17. Amarapurkar DN, Patel ND, Rane P, et al. Do different hepatitis C virus genotypes behave differently? Trop Gastroenterol. 2007;28(3):99-104.

18. Kumar D, Malik A, Asim M, et al. Response of combination therapy on viral load and disease severity in chronic hepatitis C. Dig Dis Sci. 2008;53(4):1107-1113.

19. Lawitz E, Mangia A, Wyles D, et al. Sofosbuvir for previously untreated chronic hepatitis C infection. N Engl J Med. 2013;368(20):1878-1887. 
20. Kowdley KV, Lawitz E, Crespo I, et al. Sofosbuvir with pegylated interferon alfa-2a and ribavirin for treatment-naive patients with hepatitis C genotype-1 infection (ATOMIC): an open-label, randomised, multicentre phase 2 trial. Lancet. 2013;381(9883):2100-2107.
21. Foster GR, Pianko S, Brown A, et al. Efficacy of sofosbuvir plus ribavirin with or without peginterferon-alfa in patients With HCV genotype 3 infection and treatment-experienced patients with cirrhosis and $\mathrm{HCV}$ genotype 2 infection. Gastroenterology. 2015;149(6):1462-1470. 